\title{
LES HASHTAGS MILITANTS, DES MOTS-ARGUMENTS
}

\author{
ACTIVISTS HASHTAGS, WORDS-ARGUMENTS
}

\author{
Anne-Charlotte Husson \\ Université Paris 13 Sorbonne Paris Cité - EA 7338
}

\begin{abstract}
Résumé: Cet article prend pour objet deux hashtags militants, \#Gender et \#ThéorieDuGenre, tels qu'ils sont utilisés par le compte Twitter "Veilleur sur l'actu ». Il propose de considérer les hashtags militants comme des formes de mots-arguments, c'est-à-dire d'intégrer une perspective argumentative à l'étude des hashtags. À partir d'une étude de cas précise, l'autrice montre que les concepts d'articulation (adapté de PECHEUX, 1975) et de lignée discursivoargumentative (adapté de PAVEAU, 2006) peuvent être utilisés de manière complémentaire afin de comprendre le fonctionnement pragmatique des hashtags militants.
\end{abstract}

Mots-clés: Hashtags militants ; Mots-argument ; Articulation ; Lignée discursivo-argumentative ; Genre.

Abstract: This article focuses on two activist hashtags, \#Gender and \#ThéorieDuGenre, as used by the Twitter account "Veilleur sur l'actu". It suggests considering this type of hashtags as a form of argument-words, that is, to include an argumentative perspective to the study of hashtags. Drawing from a precise case study, the author shows that the concepts of articulation (adapted from PÊCHEUX, 1975) and discursive-argumentative lineage (adapted from PAVEAU, 2006) can be used in a complementary way in order to understand the pragmatics of militant hashtags.

Keywords: Activist hashtags; Arguments-words; Articulation; Discursive-argumentative lineage; Gender.

Le militantisme sur internet, ou militantisme « 2.0 », est un objet peu étudié par l'analyse du discours numérique et pourtant largement identifié par les utilisateurs/trices d'internet, que ce soit pour s'en revendiquer ou s'en moquer ${ }^{1}$. Ses critiques dénoncent ainsi un "militantisme hashtag " (hashtag activism) caractérisé par sa nature éphémère et peu articulé aux actions sur le terrain. Ce type de militantisme superficiel serait surtout destiné à donner aux internautes l'impression d'avoir fait quelque chose (RAICU, p. 2014). Or le débat, posé en ces termes, porte toujours sur un type bien particulier de hashtags militants, créés dans une perspective de justice sociale (MITCHELL, 2014) et issus du contexte étatsunien. Ce débat se trouve alors intrinsèquement lié auxdits combats de justice sociale : antiracisme (\#BlackLivesMatter), anti-LGBTphobies (\#LoveWins), féminisme

${ }^{1}$ Cet article est issu d'un billet de blog publié le 1er juillet 2015 sur mon carnet de recherche (HUSSON, 2015b) et d'une communication donnée lors du 1er colloque international "Approaches to Discourse Analysis» (HUSSON, 2015c). 
(\#YesAllWomen)... Cet article se propose de déplacer l'attention vers un contexte et un type différents de hashtags militants. Il s'agit des hashtags \#Gender et \#ThéorieDuGenre, tels qu'ils sont utilisés pendant le mois de juin 2015 par @_VeilleActu, un compte français militant notamment contre l'ouverture du mariage aux couples homosexuels et contre la " théorie du genre ». Les deux hashtags qui m'intéressent sont bel et bien produits dans une perspective militante mais se distinguent radicalement d'un hashtag comme \#BlackLivesMatter, utilisé 9000000 de fois sur Twitter la même année et destiné à attirer l'attention sur la mort de nombreux AfricainsAméricains aux mains de la police. Le déplacement proposé vise à sortir de l'opposition pour / contre le " militantisme hashtag " pour attirer l'attention sur le fonctionnement pragmatique des hashtags militants, que je considère comme une sous-catégorie des mots-arguments. Je définis les mots-arguments comme des mots à contenu métadiscursif dense fonctionnant comme appels à des prédiscours (PAVEAU, 2006) d'ordre argumentatif. L'objectif de cet article sera de mettre en évidence la dimension argumentative de certains hashtags et, parallèlement, de contribuer à la constitution théorique de la catégorie des mots-arguments. Dans une première partie, je donne des éléments de contexte, puisque les tweets du corpus s'inscrivent dans une polémique sur le genre qui émerge en France au début des années 2010 ; je propose ensuite une description technodiscursive du corpus en question. Dans une deuxième partie, j'analyse le fonctionnement pragmatique des hashtags/mots-arguments dans mon corpus, ce qui m'amène finalement à tirer des conclusions pour l'analyse des hashtags en général.

\section{1. \#Gender et \#ThéorieDuGenre dans la polémique sur le genre}

\subsection{Contexte}

La visibilité et la fréquence d'utilisation de ces deux hashtags sur le Twitter francophone dans les années 2010 est liée à une polémique qui émerge en 2011 et ne commence à s'essouffler qu'à partir de l'été 2014. Il s'agit d'une polémique d'un type particulier, puisqu'elle est surtout marquée par les interventions, dans l'espace public, de militant-es proclamant leur opposition à la "théorie du genre ", ou parfois au genre tout court, c'està-dire au concept issu des sciences humaines et sociales qui permet de problématiser et d'analyser les manifestations sociales de la "différence des sexes ». La polémique sur le genre prend de l'ampleur à partir de l'automne 2012 avec les débats autour du projet de loi permettant l'ouverture du mariage 
aux couples de même sexe, surnommé " mariage pour tous » et adopté en mai 2013. Ce projet était une promesse de campagne du président socialiste nouvellement élu François Hollande ; or les opposant.es au « mariage pour tous " y voient un signe de l'influence de la " théorie du genre ". Cette expression, très utilisée dans le discours antigenre et dans les médias, est issue du discours catholique sur le genre, qui constitue la matrice intellectuelle et idéologique du discours antigenre. L'Église catholique développe en effet à partir de 1995 un discours extrêmement critique envers le concept de genre, accusé de dissimuler sous des abords consensuels une tentative de couper l'être humain de sa nature sexuée, mais aussi une légitimation de l'homosexualité. C'est un des penseurs catholiques sur le genre qui utilise pour la première fois l'expression connotée très péjorativement théorie $d u$ gender (ANATRELLA, 2000). Ce syntagme se stabilise ensuite au cours des années 2000 dans le discours catholique; pendant la polémique sur le genre, on trouve surtout la forme théorie du genre, mais l'emprunt gender perdure, alors même que la traduction genre est bien installée dans la littérature académique. On peut faire l'hypothèse que le choix de gender plutôt que genre est motivé par la volonté de mettre l'accent sur la dimension étrangère du concept, soulignée par Anatrella dès 2000. Deux formes sont donc employées de manière privilégiée pour désigner l'objet de la polémique : le gender et la théorie du genre. Mais alors que l'ancrage du discours antigenre dans le discours catholique est fondamental, dans la mesure où le deuxième fournit au premier ses arguments, la référence chrétienne se perd dans le passage de l'un à l'autre, le discours antigenre se voulant aconfessionnel et étant effectivement partagé par toutes sortes de locuteurs/trices — même s'ils et elles sont généralement très conservateurs/trices.

\subsection{Un compte militant : @_VeilleActu}

Même si le dernier évènement important de la polémique (la contestation d'un dispositif de l'Éducation Nationale intitulé «ABCD de l'égalité ») intervient pendant le premier semestre de 2014 et que la mobilisation perd largement en visibilité après cela, certaines organisations et militant.es plus ou moins isolé.es continuent de s'opposer activement à la « théorie du genre ». Parmi eux figurent Les Veilleurs, un mouvement né en avril 2013 dans le sillage de La Manif Pour Tous, le principal collectif anti"mariage pour tous ", qui organise plusieurs manifestations monstres contre le projet de loi. Les Veilleurs se distinguent du reste du mouvement par leurs modes d'action : ils se revendiquent de la non-violence et organisent des 
veillées au cours desquelles sont lus des textes littéraires et politiques. Très présents sur les réseaux sociaux, leurs sympathisants affichent généralement en image de profil une photographie unique, prise la nuit, d'une bougie posée au sol. C'est le cas du compte "Veilleur sur l'actu" ", créé en juin 2013 et dont est tiré le corpus, composé de 74 tweets contenant le hashtag \#Gender ou \#ThéorieDuGenre.

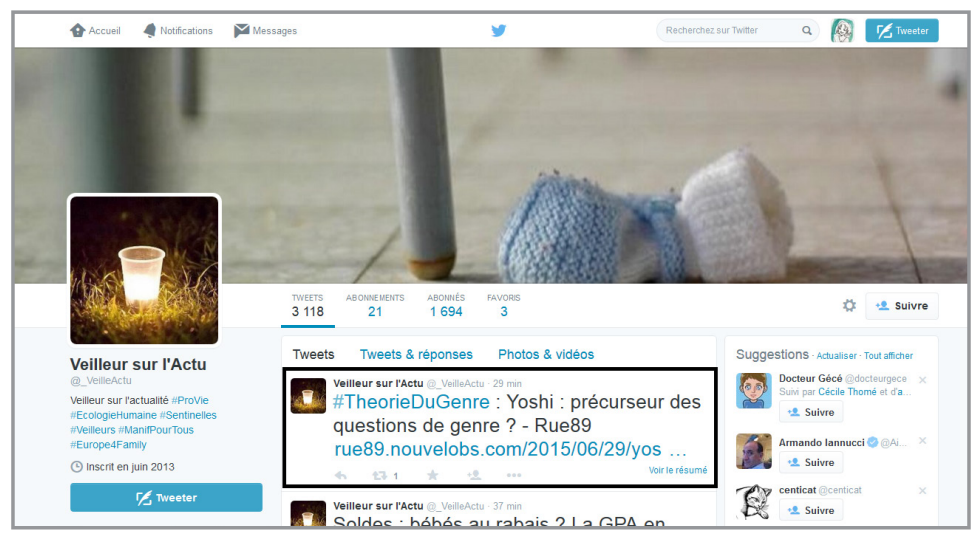

Capture d'écran 1.

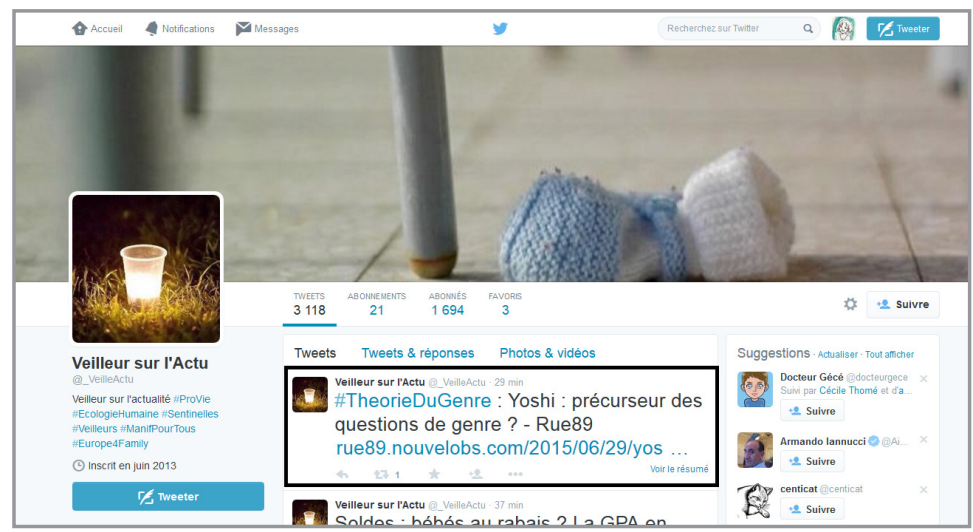

Capture d'écran 2.

${ }^{2}$ Ce compte ne se présente jamais comme un compte officiel du collectif. Le compte principal de ce dernier est @Les_Veilleurs ; s'il était toujours actif au moment cet article a été rédigé (juin 2016), le site internet (http://les-veilleurs.fr) indiqué sur son profil ne l'était plus. 
Toutes les captures d'écran correspondent au mois de collecte des données, c'est-à-dire juin 2015. On voit qu'à l'époque, le compte totalise plus de 3000 tweets et près de 1700 abonné.es; en juin 2016, le nombre de tweets avait doublé mais le nombre d'abonné.es s'était stabilisé sous la barre des 1800, confirmant donc à la fois une persistance localisée du militantisme antigenre et une baisse de l'intérêt public pour ces sujets. Malgré l'affichage aconfessionnel des Veilleurs, qu'ils partagent avec La Manif Pour Tous, le compte@_VeilleActu ne cache pas son appartenance catholique, comme on peut le voir à travers ses abonnements.

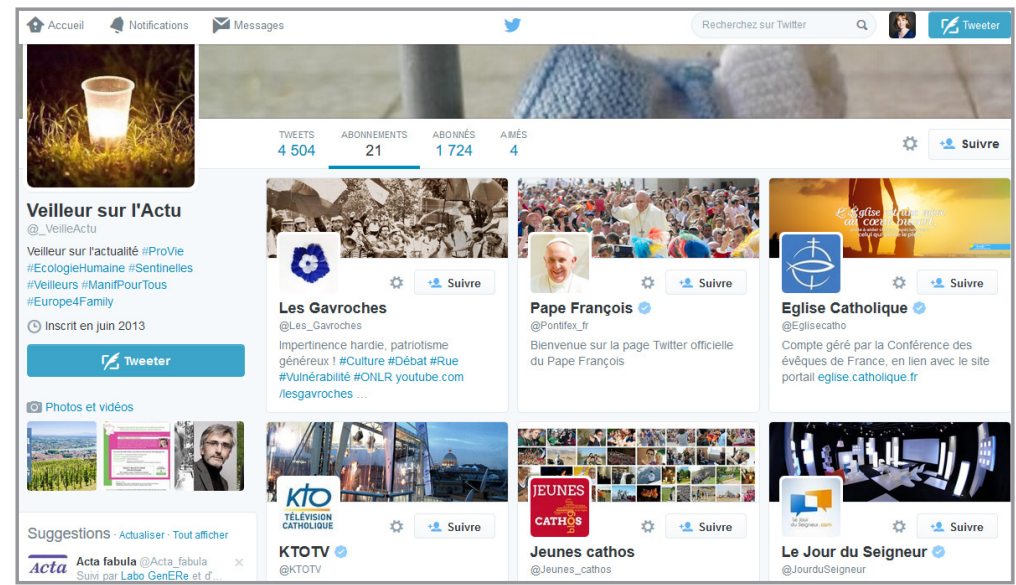

Capture d'écran 3.

Vraisemblablement tenu par plusieurs personnes, le compte publie généralement entre 2 et 15 tweets par jour. Il s'agit exclusivement de partager des articles, sous une forme fixe : le tweet comporte généralement un hashtag, presque toujours en début de tweet, le titre de l'article et un lien vers celui-ci. 


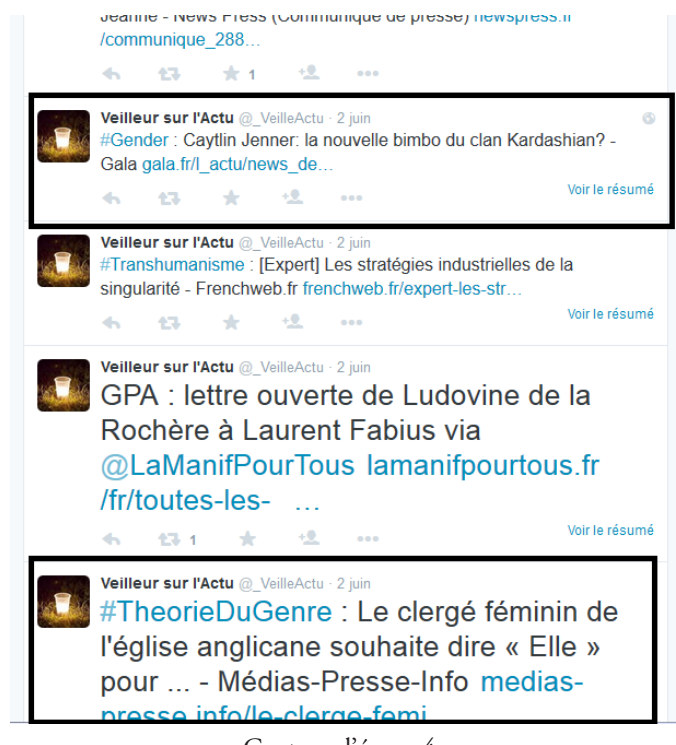

Capture d'écran 4.

Le compte n'utilise jamais la fonction retweet et joue sur le double sens de veille en français, puisqu'il se consacre exclusivement à une veille sur des sujets liés à la filiation, au mariage et à la bioéthique. Les hashtags constituent par conséquent la seule expression propre aux personnes tenant le compte, une expression à la subjectivité apparemment limitée mais, on le verra, bel et bien présente. Au cours du mois de juin 2015, les hashtags suivants sont utilisés :

\author{
\#ThéorieDuGenre \\ \#Gender \\ \#EcologieHumaine \\ \#SuicideAssisté \\ \#Euthanasie \\ \#FinDeVie \\ \#Transhumanisme, \#Transhumain \\ \#Bioéthique \\ \#AntiIVG
}


Les deux premiers, sur lesquels se concentre l'analyse, sont employés respectivement 33 et 41 fois. Ce sont les seuls qui concernent explicitement le genre, mais on remarque d'emblée que \#Gender et \#ThéorieDuGenre apparaissent contextuellement liés à des sujets de bioéthique — terme luimême " hashtagué " - comme l'euthanasie (\#SuicideAssisté, \#Euthanasie, \#FinDeVie), l'IVG (\#AntiIVG) et le transhumanisme, un mouvement prônant l'amélioration de l'espèce humaine à l'aide de la technologie. Cette association contextuelle a, déjà, valeur argumentative et n'est pas propre à ce compte, puisqu'on la retrouve régulièrement dans le discours antigenre. Pendant les débats parlementaires sur le "mariage pour tous ", le député UMP Philippe Gosselin évoque par exemple une "offensive ultra-libérale " sur les sujets suivants : " droit de la famille, politique familiale, recherche sur l'embryon, euthanasie et autres théorie du genre » ( $3^{\text {e }}$ séance du 29 janvier 2013). Mais l'association entre bioéthique et genre remonte en fait au discours catholique sur le genre. Elle est par exemple rendue explicite dans le Lexique des termes ambigus et controversés sur la vie, la famille et les questions éthiques, publié en 2003 par le Conseil pontifical pour la Famille.

On remarque aussi dans la liste ci-dessus l'emploi du terme hashtagué écologie humaine, une notion qu'on retrouve dans le corpus et qu'il est donc utile d'expliciter d'emblée. L'expression est employée pour la première fois par Benoît XVI lors d'un discours prononcé devant le Curie Romaine en décembre 2008, où elle est utilisée comme contrepoint au concept de gender :

\begin{abstract}
Il est nécessaire qu'il existe quelque chose comme une écologie de l'homme, entendue d'une juste manière. Il ne s'agit pas d'une métaphysique dépassée, si l'Eglise parle de la nature de l'être humain comme homme et femme et demande que cet ordre de la création soit respecté. Ici, il s’agit de fait de la foi dans le Créateur et de l'écoute du langage de la création, dont le mépris serait une autodestruction de l'homme et donc une destruction de l'œuvre de Dieu lui-même. Ce qui est souvent exprimé et entendu par le terme " gender ", se résout en définitive dans l'auto-émancipation de l'homme par rapport à la création et au Créateur. L'homme veut se construire tout seul et décider toujours et exclusivement tout seul de ce qui le concerne. Mais de cette manière, il vit contre la vérité, il vit contre l'Esprit créateur. Les forêts tropicales méritent, en effet, notre protection, mais l'homme ne la mérite pas moins en tant que créature, dans laquelle est inscrit un message qui ne signifie pas la contradiction de notre liberté, mais sa condition (BENOIT XVI, 2008).
\end{abstract}

On voit donc se dessiner une deuxième association notionnelle, cette fois entre genre et "écologie humaine ", et émerger des cohérences argumentatives qui demandent à être analysées. 


\section{Fonctionnement pragmatique des hashtags/mots-arguments}

\subsection{Rapprochements en contexte et dans l'interdiscours}

On a vu que le compte @_VeilleActu utilisait un nombre limité de hashtags dans ce qui, en première lecture, pourrait être interprété comme un geste de référencement - c'est la fonction de redocumentation du hashtag. Mais le choix des hashtags dans ce réservoir limité établit, d'emblée, une double cohérence argumentative, puisqu'il permet d'associer thématiquement genre, bioéthique et " écologie humaine ". Une telle cohérence, on l'a vu, est déjà présente dans l'interdiscours, entendu comme « discours-transverse » qui « traverse et connecte entre les éléments discursifs constitués par l'interdiscours en tant que préconstruit " (MALDIDIER, 1990, p. 232 ; la mise en gras est de moi). Si cette définition est bien connue, l'analyse du discours, qui s'est construite sur la triade interdiscours intradiscours - préconstruit, a cependant perdu de vue le fait que Pêcheux (suivant en cela HENRY, 1975) ne pense le préconstruit qu'en relation avec ce qu'il appelle "l'articulation d'énoncés " : c'est en effet cette dernière qui crée l'effet d'une "sorte de retour du savoir dans la pensée » (1990, p. 40). La notion, appliquée uniquement par Pêcheux et Henry au fonctionnement de la relative, est censée rendre compte de la manière dont, matériellement, l'histoire " travaille " le discours ", dans un espace "traversé de rencontres "explosives" (pas des unions de tout repos !) entre des énoncés apparemment étrangers les uns aux autres "(PECHEUX, 1981, p. 145). L’articulation d'énoncés, en tant que manifestation de l'interdiscours, mettrait en rapport l'ordre de la langue - ordre conscient, qui se manifeste par des relations de cause, de concession, etc. - et celui de l'inconscient (PECHEUX, 1975, p. 231, n. 1).

La notion d'articulation d'énoncés peut être étendue hors du cadre de la relative et mise à contribution pour rendre compte du dispositif technodiscursif propre au compte @_VeilleActu. La forme largement fixe des tweets de ce compte permet en effet de mettre en rapport un contenu extérieur (un article donné en lien et dont seul le titre est cité) avec un hashtag choisi par l'utilisateur/trice. Or si l'on reste dans la perspective d'une simple visée de redocumentation, l'association opérée entre ces deux contenus n'est pas toujours explicable au premier abord : 


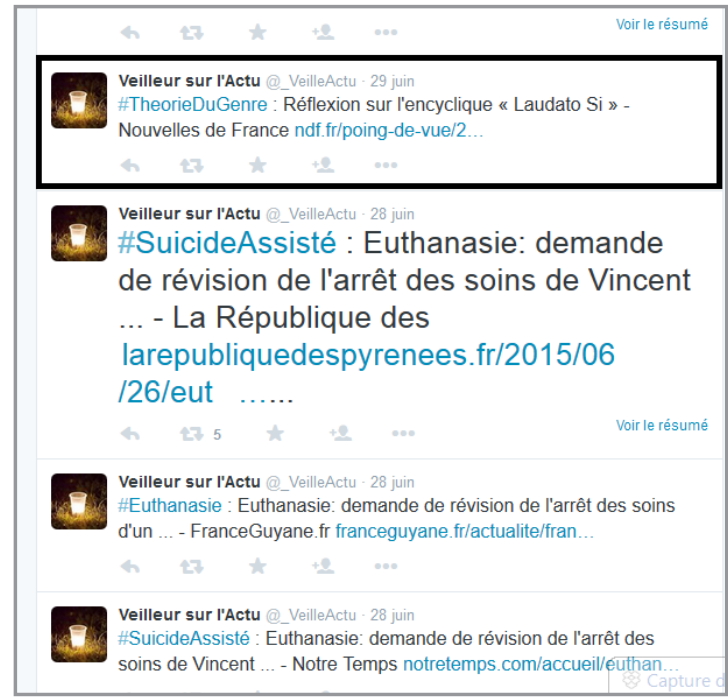

Capture d'écran 5

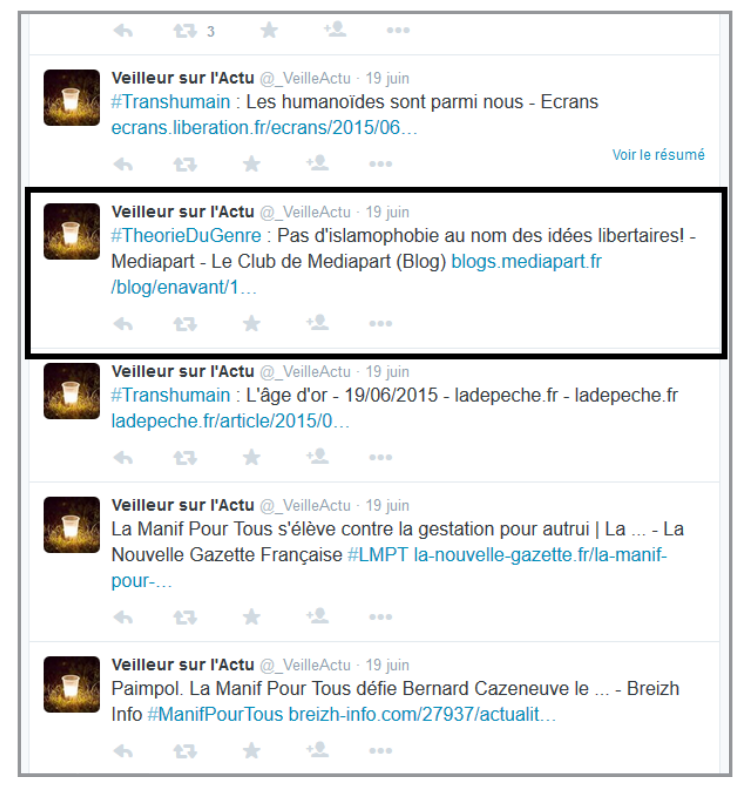

Capture d'écran 6. 
La capture d'écran $n^{\circ} 5$ présente un tweet ${ }^{3}$ renvoyant vers un article portant sur l'encyclique "Laudato $\mathrm{Si}$ » paru sur le site d'extrême droite Nouvelles de France. Publiée le 18 juin 2015 par le pape François, l'encyclique en question est consacrée aux questions environnementales et à l' "écologie humaine " prônée par son prédécesseur et censée fournir un contrepoint au concept de genre. L'articulation entre le hashtag, le titre, le lien et le contenu de l'article lui-même permet donc d'actualiser en discours une association disponible dans l'interdiscours. La capture d'écran $n^{\circ} 6$ présente une association différente, cette fois entre " théorie du genre " et islamophobie. Le lien renvoie cette fois vers un blog de Mediapart publiant une tribune sur l'islamophobie dans les milieux libertaires. Alors qu'on pouvait supposer une connivence idéologique avec l'article de Nouvelles de France, le hashtag apparaît ici comme un moyen de prendre ses distances avec le contenu de la tribune. Son auteur s'oppose à la stigmatisation des femmes voilées et dénonce au passage la "levée de boucliers contre le mariage homosexuel ou une prétendue théorie du genre », comparée à «la justification de l'esclavage ». Employé à la fois comme moyen de prise de distance et de prise de position, sans autre commentaire, le hashtag est suffisamment ambigu pour laisser une marge d'interprétation et autoriser une troisième hypothèse : celle de l'actualisation en discours d'un lien entre la politique du gouvernement socialiste, la promotion de la "théorie du genre » et celle de l'islam. Ce lien, qui fait partie de la rhétorique habituelle de l'extrême droite depuis 2012, explique le succès du mot islamogauchisme (FAURE ; DURUPT, 2016) ; il explique également la présence dans le corpus de 9 tweets renvoyant vers des articles portant sur l'islam. Dans le cas présent, l'articulation entre le hashtag et le reste du tweet constitue une de ces rencontres " explosives " dont parle Pêcheux entre des énoncés " apparemment étrangers les uns aux autres", produits d'énonciations distinctes; elle permet également de matérialiser des éléments relevant du préconstruit.

L'articulation opérée par ces tweets joue également un rôle dans la production discursive du sens des unités linguistiques gender et théorie $d u$ genre. Le deuxième constitue une formule, donc un objet de conflit et de négociation permanente exprimés dans les métadiscours des locuteurs/trices; son sens est par conséquent, si l'on suit Krieg-Planque (2009), étroitement dépendant de ces opérations métadiscursives et susceptible en permanence d'évoluer. Mais c'est aussi le cas de genre et gender, qui font, de même, l'objet d'opérations métadiscursives de la part des locuteurs/trices prenant part à la polémique : reformulation, commentaire, définition... (HUSSON, 2015a). Dans les deux cas se pose la question de la nomination. Alors que les

\footnotetext{
${ }^{3}$ Les tweets du corpus sont encadrés en noir.
} 
recherches sur le genre, à l'époque de la polémique bien engagées sur la voie de l'institutionnalisation dans l'Université française, parlent de genre pour désigner leur objet et d'études genre, études de genre ou encore études sur le genre pour se désigner elles-mêmes, le discours antigenre, on l'a déjà signalé, considère avec méfiance le terme genre et englobe généralement les recherches sur le genre sous l'étiquette d'une théorie du genre discréditée comme étant ascientifique. L'analyse du discours dispose notamment des notions de bataille sémantique et de bataille de désignation ${ }^{4}$ pour rendre compte de ces conflits portant spécifiquement sur la référence et la nomination; elle fait également un usage extensif du paradigme dialogique pour penser ces questions ${ }^{5}$. Ces perspectives gagneraient à être mises en rapport avec les notions de conflit de cadrage ${ }^{6}$ et de lutte pour la signification ${ }^{7}$ utilisées par la sociologie et les cultural studies pour rendre compte des manifestations discursives de conflictualités sociales et de la production du sens en contexte conflictuel. Or l'articulation opérée par les tweets de @_VeilleActu participe d'une production discursive du sens :

\footnotetext{
${ }^{4}$ "Est appelée ici "bataille de désignation", la lutte par laquelle, face à une même réalité qui constitue un enjeu du débat, chacun des deux camps antagonistes cherche à imposer ses dénominations contre celles de l'adversaire [...]. "Bataille sémantique" désigne plutôt la lutte que suscitent les mots communs aux deux camps lorsque les débatteurs s'appliquent à faire triompher leur sens et leur définition, contre le sens et la définition antagonistes " (RENNES, 2005, p. 503).

${ }^{5}$ Ce paradigme est invoqué pour rendre compte de rapports contradictoires à une même " réalité ». Pour Veniard 2013, l'acte de nommer tel qu'il s'exerce en contexte polémique met en évidence le fait que le langage n'est pas transparent aux choses.

${ }^{6}$ " Par conflit de cadrage on entend le conflit en tant qu'il porte sur la définition même des paramètres qui servent à le définir, à situer ses enjeux, à caractériser les intérêts et les groupes pertinents, donc à définir les acteurs" (MORMONT, 2006, \$27).

${ }^{7}$ Cette notion est destinée à rendre compte du "pouvoir idéologique [...] de signifier les évènements [to signify events] d'une façon particulière ": le réel est en effet le site d'une lutte entre des définitions antagonistes, c'est-à-dire de "politiques de la signification » (HALL, 1982, p. 69-70, ma traduction).
} 


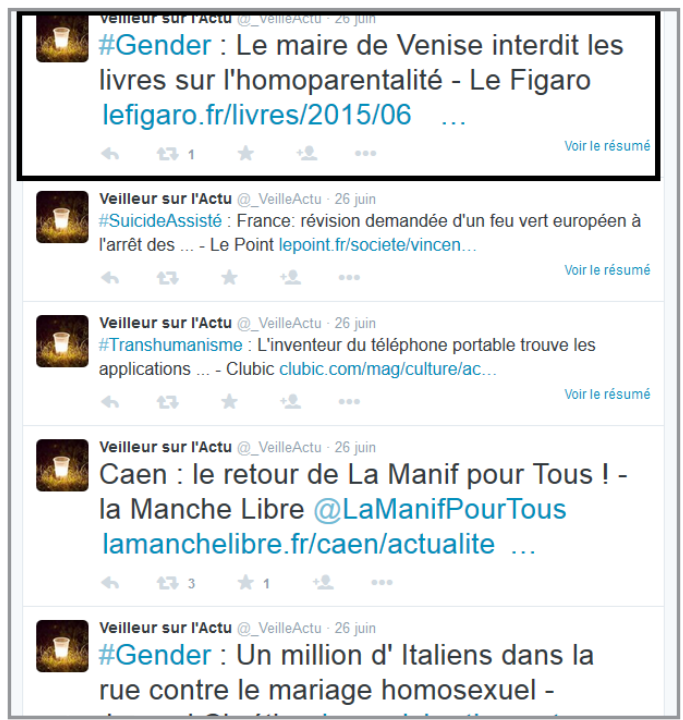

Capture d'écran 7

E. Veilleur sur l'Actu @_VeilleActu - 20 juin théorie indique que nous pourrions être. - Atlantico.fr atlantico.fr/decryptage/ino $\cdots$ $4=+7=1-1+2=0$ Veilleur sur l'Actu @ VeilleActu - 20 juin

ㄹ. \#Gender : Kristen Stewart, Miley Cyrus, Ruby Rose... la bisexualité féminine ... Atlantico.fr atlantico.fr/decryptage/kri $42^{3} 1+2 \ldots$

Veilleur sur l'Actu@_VeilleActu - 20 juin

101. \#Transhumain : Figeaccueil : le plein d'activités - ladepeche.fr ladepeche.fr/article/2015/0.

Veilleur sur l'Actu@_VeilleActu - 20 juin

Encyclique du pape François : monseigneur Boulanger livre son ....

Tendance Ouest@SOSCdOrient tendanceouest.com/actualite-1079.

Veilleur sur l'Actu@_VeilleActu - 20 juin

\#EcologieHumaine Encyclique du pape François : monseigneur

Capture d'écran 8. 
Dans les deux tweets ci-dessus, les articles partagés portent sur la sexualité (plus précisément, sur l'homosexualité et la bisexualité), mais ils sont articulés au genre grâce au hashtag \#Gender. Une telle articulation s'explique par le sens donné à genre et théorie du genre dans le discours antigenre : ils sont en effet assimilés presque systématiquement à la sexualité, plus précisément à l'homosexualité, si bien que les trois termes en viennent à se recouper largement. Ici encore, l'association n'est donc ni inédite, ni propre à @_VeilleActu mais présente en tant que préconstruit dans le discours antigenre. Elle remonte aux premiers temps de la réaction catholique au genre, où l'Église catholique identifie, dans le langage adopté par la $4^{\text {ème }}$ Conférence sur les Femmes de l'ONU (PEKIN, 1995), des " termes ambigus » qui " pourraient être compris comme approuvant les relations sexuelles en-dehors du mariage hétérosexuel ". Elle fait inclure dans le rapport final une déclaration affirmant qu'elle ne peut " accepter la terminologie ambiguë concernant la maîtrise absolue de la sexualité et de la fécondité, en particulier parce qu'elle pourrait être interprétée comme signifiant que la société approuve l'avortement et l'homosexualité " (ONU, 1995). À ce texte est associé une "Déclaration interprétative " portant sur le terme gender, dont le Saint-Siège affirme qu'il " procède de la distinction biologique entre l'homme et la femme " et ne doit pas contribuer à des "vues répandues dans le monde selon lesquelles l'identité sexuelle peut être adaptée indéfiniment à des fins nouvelles et différentes " (HUSSON, 2015a; SCHWEBEL, 2015). Le terme genre est considéré, pour résumer, comme une sorte de cheval de Troie risquant de mener à l'acceptation de l'homosexualité. L'existence de cette association / confusion entre genre, sexualité et homosexualité dans le discours catholique sur le genre explique que le projet de loi ouvrant le mariage aux couples de même sexe ait été interprété par ses opposant.es comme une conséquence de l'influence de la "théorie du genre " sur le pouvoir politique et législatif français. Comme dans le cas de l' " écologie humaine " et de l'islam, on est donc en présence de plusieurs phénomènes discursifs distincts mais liés : une association disponible dans l'interdiscours et d'un effet de préconstruit se concrétisant par l'articulation d'énoncés, ainsi qu'une (re)production, en discours, de cette même association et donc du sens de gender et théorie du genre.

\subsection{Une fonction argumentative des hashtags}

Il est donc évident que le rôle d'opérateur de redocumentation, s'il existe, est loin d'être la fonction première des hashtags utilisés par le compte 
@_VeilleActu. Si l'on se place dans la perspective de Zappavigna (2011), les hashtags permettraient de créer une " affiliation diffuse ", c'est-à-dire de constituer des communautés virtuelles via la fonction d'investigabilité (PAVEAU, 2013). Les hashtags permettent donc aux utilisateurs/trices de former des communautés d'intérêt plus ou moins lâches et en reconfiguration permanente. Cette notion peut être appliquée avec profit, me semble-t-il, à l'étude du militantisme en ligne; elle permet d'aller au-delà de l'idée du rôle organisationnel des hashtags, intéressante pour les sciences de l'information et de la communication mais n'offrant pas, en soi, de prise à l'analyse du discours numérique. En l'occurrence, les hashtags utilisés par@_VeilleActu permettent d'inscrire le compte dans le réseau de la militance antigenre en ligne et dans le discours antigenre. Cette opération d'affiliation permet, dans le même temps, de marquer un positionnement énonciatif étroitement lié au dispositif technodiscursif et demandant, là aussi, à être analysé en termes argumentatifs.

Ce que j'ai pour l'instant décrit en termes d'articulation peut également être analysé, de manière complémentaire et dans une perspective argumentative, comme une forme d'inscription dans une lignée à la fois discursive et argumentative, inscription permise par les mots à contenu métadiscursif dense que sont les mots-arguments. Plus dynamique que la notion de répertoire d'arguments, celle de lignée discursivo-argumentative permet de rendre compte de l'ancrage de l'antériorité dans des unités lexicale qui font signe, à leur tour, vers cette antériorité. Cette notion s'appuie sur le retravail du préconstruit et de la mémoire discursive par Paveau (2006, p. 118), qui propose d'appeler prédiscours « un ensemble de cadres prédiscursifs collectifs (savoirs, croyances, pratiques)" donnant "des instructions pour la production et linterprétation du sens en discours. Dans sa perspective, les « lignées discursives " sont des " configurations sémantiques portées par les discours transmis " (PAVEAU, 2006, p. 123). Dans le prolongement de ce travail, je considère pour ma part les mots-arguments comme des " appels aux prédiscours » et des points d'inscription de lignées discursivoargumentatives. Ils condensent donc un contenu sémantico-argumentatif préalable et partagé par les membres d'une communauté discursive, en l'occurrence, les militant.es antigenre. Une telle conception met en jeu, comme le préconstruit, une théorie du sujet: pour Pêcheux, qui suit en cela Althusser, le préconstruit " correspond au "toujours-déjà là" de l'interpellation idéologique qui fournit-impose la "réalité" et son "sens" sous la forme de l'universalité (le "monde des choses") »; il est inséparable de l'articulation, autre type de "décalage", qui «constitue le sujet dans son rapport au sens, de sorte qu'elle représente, dans l'interdiscours, ce qui détermine 
la domination de la forme-sujet "(1975, p. 229, ital. dans l'original). La perspective de Paveau (idem) est différente et plus optimiste : il ne s'agit plus de rendre compte de l'assujettissement nécessaire du "sujet-parlant " mais des dimensions perceptive et cognitive de l'antériorité du sens. Au lieu de se concentrer sur les « évidences universelles » du sens, elle met l'accent sur le contenu sémantique des données prédiscursives collectives et restaure donc une certaine agentivité pour les locuteurs/trices à l'égard de la production discursive du sens.

Ce détour par les théories du préconstruit permet de comprendre le double mouvement de l'articulation dans les tweets du corpus. Le dispositif de ces tweets, qui associe un hashtag choisi par le locuteur ou la locutrice à un contenu extérieur, repose en effet autant sur une articulation établie avant et ailleurs - dans le discours antigenre et/ou le discours catholique sur le genre - qu'il produit lui-même, en discours, une telle articulation. Autrement dit, l'articulation n'est pas seulement transmise sous la forme d'une lignée discursivo-argumentative s'inscrivant dans un motargument : l'emploi du mot-argument permet de renouveler et d'affirmer une telle articulation. en outre, les hashtags/mots-arguments sont plus que des opérateurs de positionnement énonciatif : leur emploi dans ces tweets est ce qui permet la production et la reconfiguration permanente de l'identité associée au compte ${ }^{8}$. En plus de mettre en jeu la nomination (genre, gender, théorie du genre) et l'extension de l'objet de la polémique (quels domaines sont-ils touchés par le genre ?), cette pratique questionne en effet le rapport entre énoncé, sujet et positionnement énonciatif. La pratique du " hashtagage " peut ainsi être considérée, in fine, en termes de performance identitaire : son étude, pour l'analyste du discours polémique que je suis, suppose alors de ne pas me contenter d'appliquer mon propre métadiscours et mes catégories analytiques sur mon objet, mais de me situer au niveau des pratiques et de l'évènement que constitue l'énonciation (GUILHAUMOU; MALDIDIER, 1986). Cela conduit à mettre de côté l'articulation entre idéologie, préconstruit et assujettissement pour retrouver une agentivité possible pour le sujet dans sa manière de mobiliser les prédiscours - et ce, sans retomber pour autant dans l'écueil d'un sujet tout-puissant, maître de son dire. Un tel projet trouve un appui solide dans une autre théorie de l'articulation, formulée initialement pour les cultural studies par Laclau (1977) et développée par Hall ([1998] 1996). Le premier cherche à rendre compte du caractère non-nécessaire de l'articulation entre concepts et domaines idéologiques, c'est-à-dire à rompre avec l'idée des

\footnotetext{
${ }^{8}$ Cf. à ce sujet la critique formulée par Guilhaumou (2011) à l'égard de la notion de positionnement énonciatif telle qu'elle est maniée par Maingueneau.
} 
classes comme blocs homogènes produisant des discours univoques. Les articulations, explique-t-il, se font et se défont, à l'image de l'articulation entre conservatisme et absolutisme, longtemps dominante dans les régimes monarchiques européens. Hall développe et modifie cette théorie en gardant toujours à l'esprit l'impératif d'une théorie orientée par la pratique, c'est-à-dire destinée in fine à faciliter l'intervention sociale. La théorie de l'articulation est, selon lui, ce qui « nous permet de penser la façon dont l'idéologie donne du pouvoir aux individus, en les rendant capables de donner du sens ou de l'intelligibilité à leur situation historique, sans réduire ces formes d'intelligibilité à leur localisation socio-économique ou de classe ni à leur position sociale " ([1986] 1996, p. 142). De cette théorie, je retiens pour les besoins de cet article deux choses : d'abord, le caractère nonnécessaire - et en même temps puissamment contraignant — de certaines articulations ; ensuite et surtout, l'idée d'une agentivité des locuteurs/trices, qui peuvent mobiliser ces articulations pour faire sens de leur situation historique et politique.

Il s'agira donc in fine d'identifier, dans les configurations technodiscursives du corpus, ce qui relève d'une possible agentivité des locuteurs/trices dans leur mobilisation d'articulations définies ailleurs et avant eux, et de comprendre dans quelle mesure cela peut relever d'une performance identitaire, alors que le dispositif choisi semble effacer toute subjectivité. Je prends pour exemple l'opération de réorientation sémantique et argumentative effectuée via le hashtag par rapport au contenu du lien partagé. 


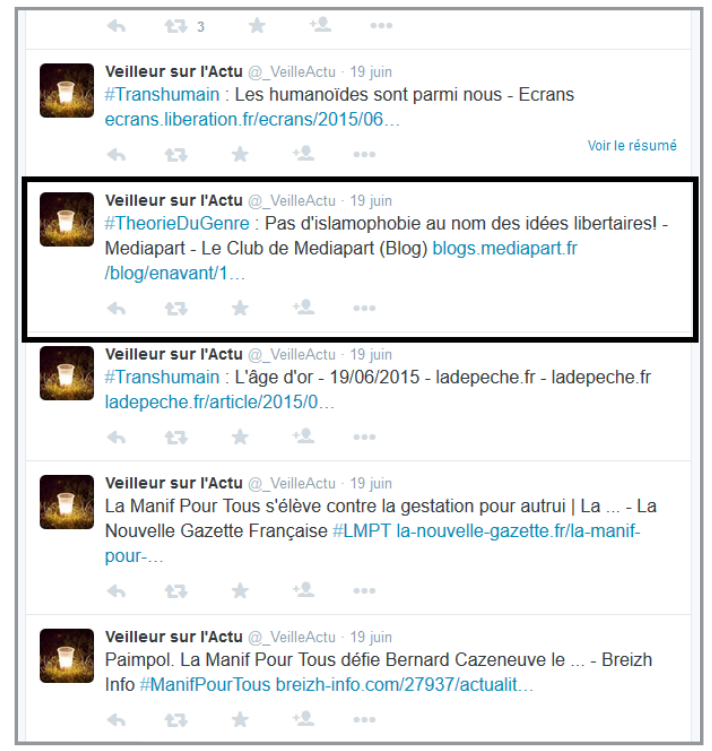

Capture d'écran 9.

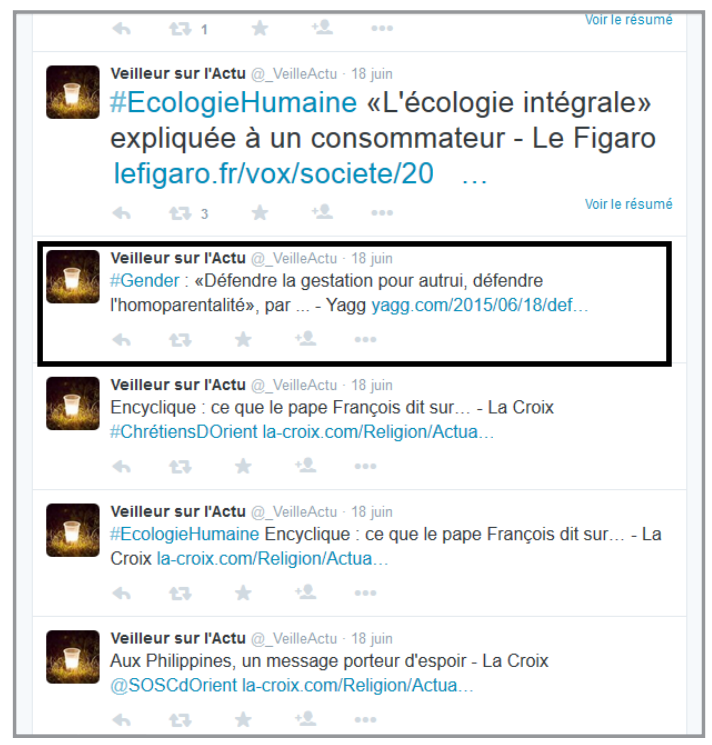

Capture d'écran 10. 


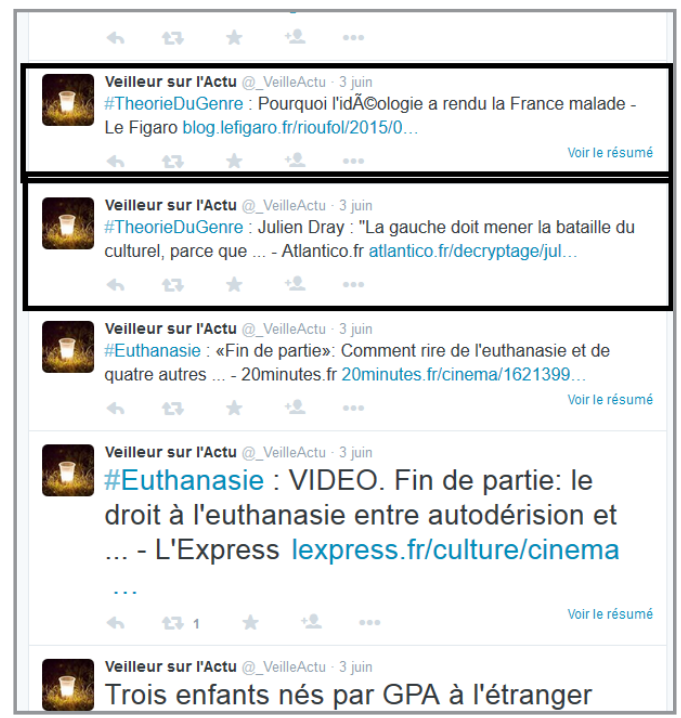

Capture d'écran 11.

Les deux premiers tweets proviennent de médias explicitement positionnés à gauche, contrairement aux sources généralement citées par le compte, comme Le Figaro, Valeurs Actuelles ou encore La Croix. Yagg est en outre un média communautaire LGBT. Quant au troisième tweet, bien que publié sur le "pure player" de droite Atlantico, il présente une interview du député PS Julien Dray. Dans tous les cas, le hashtag a une fonction polémique, puisque l'opération de labellisation entre directement en conflit avec le positionnement explicite des articles partagés. Les auteurs des deux premiers articles évoquent rapidement, pour le discréditer, le militantisme antigenre et rejettent l'expression théorie du genre; le geste polémique du hashtagage revêt donc, dans ces cas précis, une dimension ironique. En revanche, l'interview de Julien Dray n'évoque à aucun moment le genre. L'attribution du hashtag \#ThéorieDuGenre relève donc en l'occurrence d'un geste purement interprétatif de la part du locuteur ou de la locutrice, un geste qui oriente, pour la personne cliquant sur le lien, la lecture de l'article. Une orientation similaire a lieu quand @_VeilleActu partage, à deux reprises, des articles portant sur le scandale créé par Rachel Dolezal, une militante antiraciste étatsunienne dont on découvre en juin 2015 qu'elle se colore la peau artificiellement depuis plusieurs années pour se faire passer pour une personne de couleur, alors qu'elle est en réalité blanche. 


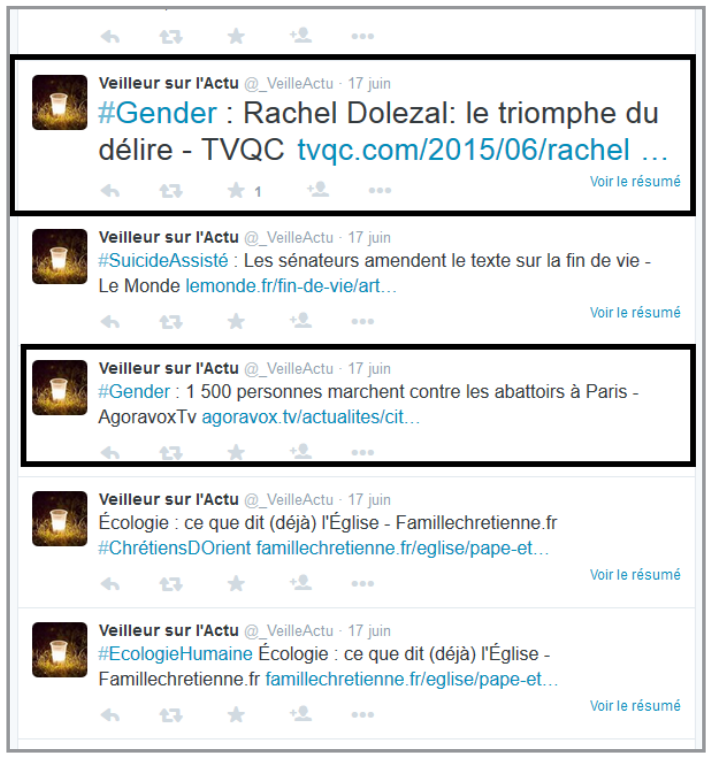

Capture d'écran 12.

\begin{tabular}{|l|}
\hline Veilleur sur l'Actu @_VilleActu - 13 juin \\
\#heorieDuGenre : Les réacs de gauche - Politis politis.fr/Les-reacs- \\
de-g...
\end{tabular}

Capture d'écran 13. 
L'attribution du hashtag, qui plus est en début de tweet, a ici pour effet d'établir une comparaison entre le fait de se colorer la peau afin de se faire passer pour noire et celui d'être transgenre : dans les deux cas, il s'agirait de se faire passer pour ce que l'on n'est pas. Cette articulation créée par le tweet repose en outre sur une autre lignée discursivo-argumentative du discours antigenre, celle du choix et de la libre détermination d'un individu affranchi de sa nature sexuée.

\section{Conclusion}

En conclusion, les hashtags militants analysés ne sont ni des descriptions univoques du contenu des liens partagés, ni, encore moins, de simples opérateurs de redocumentation. La notion d'affiliation diffuse, quoiqu'elle puisse être appliquée avec profit au militantisme en ligne, ne suffit pas non plus à expliquer ce qui est en jeu ici car elle ne revêt pas de dimension argumentative. Les hashtags militants du corpus sont des métadiscours et des mots-arguments aux fonctionnements complexes et multiples, fonctionnements qu'on peut rassembler en deux groupes. D'abord, ils jouent un rôle dans la production du sens discursif des unités linguistiques gender et théorie du genre et mettent en question le processus de nomination, dans la mesure où ils constituent des étiquettes polémiques. En outre, ils mettent en jeu des processus qui ont à voir avec le dispositif de Twitter lui-même : non seulement ils permettent, au sein du tweet, une (ré)orientation du contenu du lien, mais ils ont également un effet réflexif sur l'identité du compte et demandent alors à être analysés comme gestes interprétatifs et producteurs de positionnements énonciatifs. La notion d'articulation permet de rendre compte de ces deux versants de la pragmatique des hashtags/mots-arguments. Cette notion peut être comprise, au-delà de sa théorisation originelle dans le cadre de la proposition relative, comme ce qui manifeste l'hétérogénéité et la crée tout à la fois. Elle met en jeu l'ordre conscient et matériel de la langue (l'articulation des séquences discursives) et ce qui la travaille de l'extérieur (dans le cas de cette étude, les prédiscours et les lignées discursivo-argumentatives).

\section{Références}

ANATRELLA, Tony. À propos du concept de " gender ». Revue d'éthique et de théologie morales, n. 2015, p. 29-74.0 2000. 
BENOIT XVI. Discours du pape Benoît XVI à la Curie Romaine à l'occasion de la rencontre traditionnelle des vœux de Noël. 22 décembre 2008.

FAURE, Sonya et DURUPT, Frantz. Islamo-gauchisme, aux origines d'une expression médiatique. Libération, 14 avril 2016.

GROSSBERG, Lawrence; HALL, Stuart. On Postmodernism and Articulation : An Interview with Stuart Hall. Stuart Hall. Critical Dialogues in Cultural Studies, Morley Davis. et Chen Kuan-Hsing (éds), Londres et New York, Routledge, p. 131-150. [1986] 1996.

GUILHAUMOU, Jacques; MALDIDIER, Denise. De l'énonciation à l'évènement discursif en analyse du discours. Histoire Épistémologie Langage, v. 8, n. 2, p. 233-242.

GUILHAUMOU, Jacques. Où va l'analyse du discours? Autour de la notion de formation discursive. Marges linguistiques, n. 9, p. 95-114. 2005.

HALL, Stuart. The rediscovery of "ideology " : return of the repressed in media studies. Culture, society and the media, Gurevitch Michael. et al. (Éds), Londres et New York, Methuen, p. 56-90, 1982.

HENRY, Paul. Constructions relatives et articulations discursives. Langages, v. 9, n. 37, p. 81-98. 1975.

HUSSON, Anne-Charlotte. Stratégies lexicales et argumentatives dans le discours antigenre. Sextant, n. 31, p. 93-108. 2015 a.

. \#ThéorieDuGenre, \#Gender: deux hashtags à rôle argumentatif. (Dis)cursives [carnet de recherche], 2015b. Disponible à l'adresse $:<\underline{\text { http:// }}$ cursives.hypotheses.org/170>. Dernier accès le : 20 juin 2016.

Hashtags as "mots-arguments ": The controversy around gender in 2010's France. Communication présentée lors du 1er Colloque international "Approaches to Digital Discourse Analysis ", Valence (Espagne), novembre 2015c.

KRIEG-PLANQUE, Alice. La notion de " formule " en analyse du discours. Cadre théorique et méthodologique. Besançon, Presses Universitaires de Franche-Comté, 2009.

LACLAU, Ernesto. Politics and Ideology in Marxist Theory. Capitalism - Fascism - Populism. Londres: NLB, 1977. 
MALDIDIER, Denise. L'inquiétude du discours. Textes de Michel Pêcheux, choisis et présentés par Denise. Maldidier. Paris: Editions des Cendres, 1990.

MITCHELL, Tasha. Top 10 Social Activism Hashtags of 2014. The Huffington Post. 17 décembre 2014. Disponible à l'adresse : < $\underline{\text { http://www. }}$ huffingtonpost.com/tasha-mitchell/top-10-social-activism-ha b 6331648. $\underline{\mathrm{html}}>$. Dernier accès le : 20 juin 2016.

MORMONT, Marc. Conflit et territorialisation. Géographie, économie, société, v. 8, n. 3, p. 299-318. 2006. Disponible à l'adresse : <http://www. cairn.info/revue-geographie-economie-societe-2006-3-page-299.htm >. Dernier accès le : 20 juin 2016.

ORGANISATION DES NATIONS-UNIES. Déclaration et Programme d'action de Beijing. 15 septembre 1995.

PAVEAU, Marie-Anne. Les prédiscours. Sens, mémoire cognition. Paris, Presses Sorbonne Nouvelle, 2006.

. Hashtag. Technologies discursives. 22 mai. 2013. Disponible à l'adresse : <http://technodiscours.hypotheses.org/488>. Dernier accès le : 20 juin 2016.

PÊCHEUX Michel. Les vérités de la Palice. Paris: Maspero, 1975.

. L'énoncé : enchâssement, articulation et déliaison. In: COURTINE Jean-Jacques. [et al]. (Éds). Colloque " Matérialités discursives». Lille : Presses universitaires de Lille, 1981. p. 143-148.

RENNES, Juliette. Le mérite et la nature. Une controverse républicaine : la mixité du prestige professionnel (1880-1940). Thèse de doctorat, 2005.

RAICU, Irina. Hashtag Activism and the Power of Attention: An Ethics Case Study. Markkula Center for Applied Ethics. $1^{\text {er }}$ mai 2014. Disponible à l'adresse : <https://www.scu.edu/ethics/focus-areas/internet-ethics/ resources/hashtag-activism-and-the-power-of-attention $>$.. Dernier accès le : 20 juin 2016.

SCHWEBEL, Joke. Recognizing Gender and Sexuality at the United Nations. Sextant, n. 31, p. 25-41. 2015. 
VENIARD, Marie. Nommer un événement : le désigner et/ou le signifier ? Le cas de la guerre en Afghanistan (2001) dans Le Monde et Le Figaro. Les Mots de la guerre : imaginaires, langages, représentations, Puccini Paola et Regattin Fabio (Éds), Bologne, CLUEB, p. 27-41. 2013.

ZAPPAVIGNA, Michele. Ambient affiliation: A linguistic perspective on Twitter. New media and society, v. 13, n. 5, p. 788-809. 2011.

Recebido em: 30/07/2016

Aceito em: 25/10/2016 